\title{
Soft tissue involvement, mediastinal pseudotumor, and venous thrombosis in pustulotic arthro-osteitis
}

\author{
A study of eight new cases \\ Marnix van Holsbeeck, M.D. ${ }^{1}$, William Martel, M.D. ${ }^{2}$, Jan Dequeker, M.D. ${ }^{3}$, Alex Favril, M.D. ${ }^{1}$, \\ Jan Gielen, M.D. ${ }^{1}$, Johny Verschakelen, M.D. ${ }^{1}$, and Michel Hoogmartens, M.D. ${ }^{4}$ \\ Departments of ${ }^{1}$ Radiology, ${ }^{3}$ Rheumatology, and ${ }^{4}$ Orthopedics, University Hospitals K.U. Leuven, Leuven, Belgium \\ 2 Department of Radiology, University of Michigan Medical Center, E. Medical Center Drive, Ann Arbor, Michigan, USA
}

\begin{abstract}
A syndrome of hyperostosis of the thoracic wall, nonspecific signs of inflammatory disease, and palmar and plantar pustulosis is described in eight patients (Table 1). Seven had intersternocostoclavicular ossification [12], and one had chronic recurrent multifocal osteomyelitis [2]. This complex of findings has been called "pustulotic arthro-osteitis" $[5,12]$. This report emphasizes the periosseous soft tissue inflammation and the unexplained subclavian and mediastinal vein thrombosis seen in two patients [8]. Inflammatory periosseous and mediastinal lesions were seen on plain films in all eight patients and on computed tomographic (CT) scans in seven. Radiographs of the spine showed a spondyloarthropathy in three patients. This was characterized by ossification of the vertebral ligaments and sclerosis of the vertebral bodies. Awareness of the radiologic features of pustulotic arthro-osteitis is important because the clinical, biochemical and pathologic findings are often nonspecific and misleading $[5,8,12]$.
\end{abstract}

Key words: Palmoplantar pustulosis - Chronic recurrent multifocal osteomyelitis - Sternocostoclavicular hyperostosis - Clavicle: hyperostosis Ribs: abnormalities - Sternum : abnormalities

Intersternocostoclavicular hyperostosis and chronic recurrent multifocal osteomyelitis are recognized syndromes [2, 7-12] whose classification under a common name has been proposed by several authors in recent years $[1,5,12]$. Approximately $50 \%$ of patients with either syndrome have a skin condi-

Address reprint requests to: M. van Holsbeeck, M.D., Department of Radiology, University Hospitals, K.U. Leuven, Herestraat 49, B-3000 Leuven, Belgium tion called "palmoplantar pustulosis" (Fig. 7). Hence, some authors prefer the term "pustulotic arthro-osteitis and related disorders" [5, 12]. The findings of hyperostosis and inflammation and the multifocal character of the lesions in the two syndromes have suggested another name: "multiple inflammatory hyperostosis" [1].

It was noted early that the lesions were not strictly limited to bone. An associated enthesitis was stressed by Sonozaki et al. [12], and Kohler et al. [8] noted the thoracic outlet syndrome as a frequent complaint and thrombosis of the subclavian vein as a possible complication. In our series, two patients presented with a superior vena cava syndrome and, in one of these, there was a soft tissue mass in the anterosuperior mediastinum. We performed CT scans in seven of the eight patients to evaluate the mediastinal involvement.

These cases are commonly misdiagnosed. Three patients in this series were suspected of having a mediastinal tumor, and two were thought to have periarticular disease of the shoulder. The chest wall pain in one patient was diagnosed as coronary heart disease.

\section{Case reports}

During the past 5 years, seven cases of intersternocostoclavicular hyperostosis and one case of chronic recurrent multifocal osteomyelitis (case 5) were diagnosed at our institution.

\section{Case 1}

A 46-year-old teacher had a painful swelling in the neck for 2 months. Chest radiographs showed a retrosternal mass, which was initially misdiagnosed as an enlarged thyroid gland, displacing the trachea to the left. On examination, no thyroid enlargement was present. There was venous engorgement in the neck with dilated collateral veins in the upper right chest and shoulder and swelling of the right arm. The erythrocyte sedimentation rate (ESR) was raised at $62 \mathrm{~mm} / \mathrm{h}$. White cell 

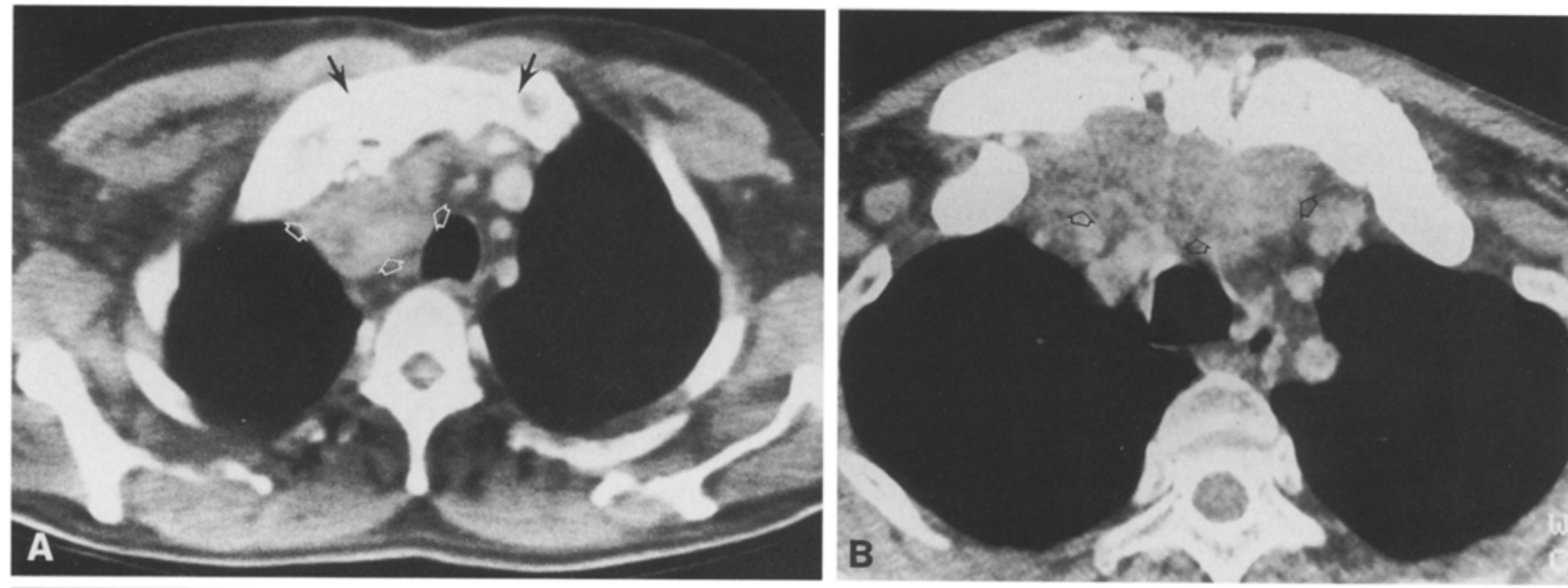

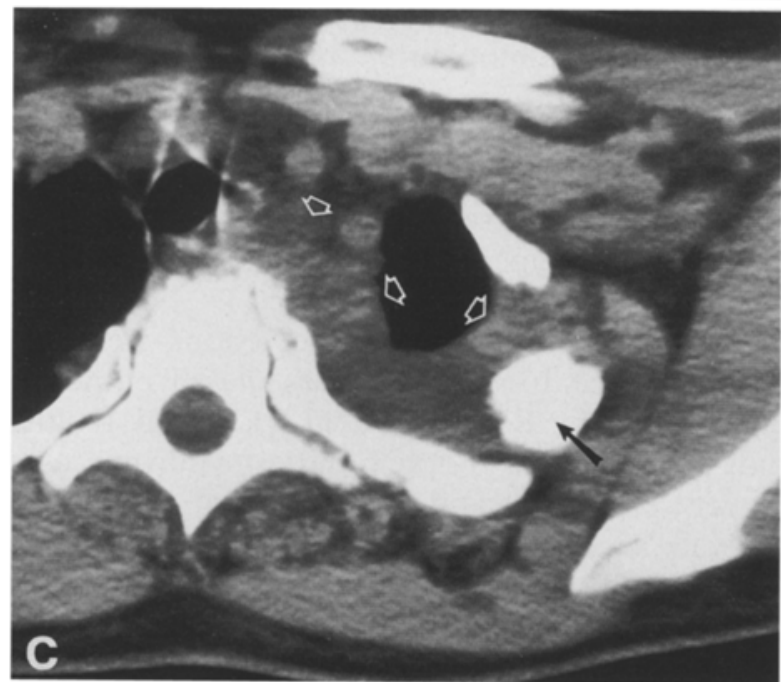

Fig. 1 A-D. Soft tissue mass or soft tissue infiltration around the hyperostotic bones was found in all our patients. In three patients this soft tissue component had a tumor-like appearance. This figure illustrates the variety of soft tissue involvement in our patient group. A Case 1. Contrast-enhanced CT scan of the upper mediastinum. First rib synostosis with the sternum (black arrows) best shown by bony windows. Contrast enhancement of retrosternal soft tissue mass. The thrombosed innominate vein is located within the mass (open arrows). B Case 2. Contrast-enhanced CT scan of the upper mediastinum. There is diffuse infiltration of fat and displacement of vessels by a large soft tissue mass in the superior mediastinum (open arrows). The posterior sternoclavicular junction is irregular. C Case 5. CT scan of the left lung apex showing hypertrophied 2nd left rib (black arrow). Pleural thickening (open arrows) extends into the posterior mediastinum). D Case 7. CT scan of the upper mediastinum showing hypertrophied manubrium sterni (black arrow). Retrosternal fat infiltration (open arrows) was seen in all patients in whom CT was performed

count and thyroid function were normal. CT showed a soft tissue mass in the upper right anterior mediastinum; this mass was initially thought to be tumor or lymphadenopathy

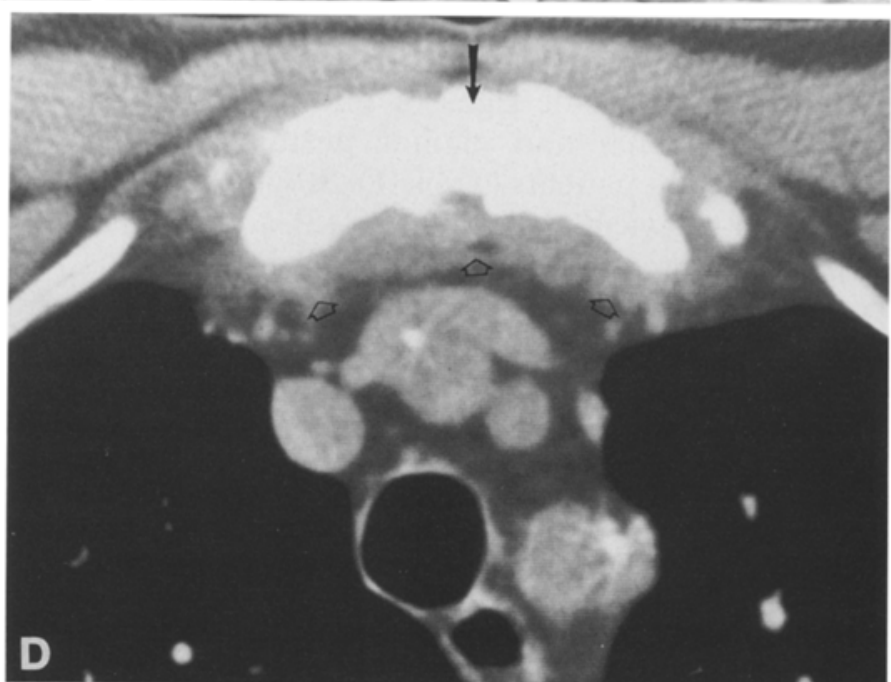

(Fig. 1A). There was thrombosis of the right subclavian and innominate veins. Conventional tomograms showed hyperostosis and sclerosis of the medial portions of the right clavicle and first rib.

Venography showed occlusion of the right cephalic, internal jugular and subclavian veins, and multiple collateral veins in the upper right chest wall and neck (Fig. 2). There was an edematous mass in the upper mediastinum at surgery. Histologic examination showed nonspecific chronic inflammation.

The thrombosis was thought to be due to venous obstruction by the mediastinal mass or to restriction of the thoracic outlet by bony enlargement. Anticoagulant treatment (Marcoumar $1 / 2$ tablet daily) was effective, but after therapy was stopped, the thrombosis recurred. Partial resection of the first rib was beneficial. CT showed no change in the mediastinal mass.

Two years later the patient had frequent episodes of upper right chest and shoulder pain unrelated to exertion, sometimes accompanied by a pustular skin rash on the palms of his hands and the soles of his feet. Plain films showed hyperostosis of the medial portions of the right clavicle and first rib with ossification of the costoclavicular ligament and local soft tissue swelling, all of which suggested intersternocostoclavicular hyperostosis. The symptoms responded to anti-inflammatory drugs, and there was no further recurrence. 


\section{Case 2}

A 53-year-old man complained of retrosternal pain, which was thought to be cardiac in origin, radiating to the right upper chest and shoulder. There was bony swelling of the manubrium sterni. Pustules were present on the palms and soles. The ESR was $61 \mathrm{~mm} / \mathrm{h}$. Coronary angiograms were normal. Radiographs showed enlargement and sclerosis of the clavicles and manubrium sterni, sclerosis of the first ribs, and extensive ossification of both costoclavicular ligaments, findings consistent with intersternocostoclavicular hyperostosis (Figs. 3, 4). CT showed an infiltrating soft tissue mass in the mediastinum as well as between the sternum and the pectoralis muscle (Fig. 1 B). A biopsy of the left clavicle demonstrated new bone formation and granulation tissue. Indomethacin (100 mg daily) produced complete relief of symptoms.

\section{Case 3}

A 65 -year-old woman described a 20 -year history of recurrent painful swelling of the right shoulder, neck and lumbar back pain, and pustulosis. The ESR was elevated at $55 \mathrm{~mm} / \mathrm{h}$. Radiographs showed severe enlargement of the left clavicle and first rib and manubrium sterni with ossification in the costoclavicular ligaments. The medial end of the right clavicle was slightly hypertrophied. These findings are consistent with intersternocostoclavicular hyperostosis. Plain films of the lumbar spine showed hypertrophic ossification of the anterior longitudinal ligament, dense new bone formation in the vertebral bodies, and facet joint ankylosis (Fig. 5 A, B). An asymmetric sacroiliitis was present (Fig. 6). The patient declined further investigation.

\section{Case 4}

A 43-year-old man was referred for evaluation of an arthritis of both wrists. He gave a 10-year history of pustulosis affecting his hands and feet (Fig. 7) and also his back and buttocks. There were no signs of psoriasis.

He complained of pain in the upper chest and back, worse at night and in cold weather, associated with morning stiffness. Five years previously he had noted enlarged veins in his neck, arms, and chest. Tests for syphilis, rheumatoid factor, HLAB27 antigen, antinuclear factor, human immunodeficiency virus, and brucella agglutinins were negative. The ESR was slightly raised at $30 \mathrm{~mm} / \mathrm{h}$. The white cell count and alkaline phosphatase level were normal. Serum electrophoresis showed slightly elevated levels of alpha-2 globulins $(1.06 \mathrm{~g} / 100 \mathrm{ml})$ and gamma globulins $(1.4 \mathrm{~g} / 100 \mathrm{ml})$. The lupus anticoagulant factor was positive (ratio $1 / 64$ ).

Radiographs of the wrists showed generalized bilateral joint space narrowing. Films of the spine revealed hypertrophic ligamentous ossification and ankylosis of the facet joints. There was left-sided sacroiliitis. A chest film demonstrated a widened upper mediastinum. There were hypertrophy and sclerosis of the clavicles (Fig. 8), findings compatible with pustulotic arthro-osteitis. A technetium bone scan showed asymptomatic sites of hyperactivity in both thighs and forearms and plain films showed fusiform broadening of the femoral shafts (Fig. 9). The distal end of the radius was enlarged and sclerotic bilaterally, and there was periosteal new bone formation. A contrastenhanced CT scan of the mediastinum showed clavicular hypertrophy (Fig. 10). There were multiple unenhancing nodular structures in the mediastinum. The absence of enhancement suggests that these were enlarged lymph nodes rather than collateral veins (Fig. 10). The subclavian veins failed to fill with contrast medium, but there was contrast enhancement of azygos and accessory hemiazygos veins (Fig. 10). Nuclear magnetic resonance images showed flow in a dilated azygos vein but not in the upper part of the right innominate vein (Fig. 11). Bilateral digital subtraction venograms of the arms showed obstruction of the subclavian veins (Fig. 12).

Contrast medium injected into the right arm passed through a network of collateral veins in the neck and into the jugular veins. Contrast medium injected into the left arm passed through the accessory hemiazygos system and into the tortuous and dilated azygos vein. CT of the abdomen suggested retroperitoneal fibrosis since both ureters were dilated and there was a soft tissue mass in the retroperitoneum. The patient's symptoms resolved with indomethacin.

\section{Case 5}

A 21-year-old woman complained of neck pain of 3 years duration. The pain radiated to the left shoulder and was accompanied by fatigue and morning stiffness and pain and paresthesia in the left hand at night. The pain responded to anti-inflammatory drugs, but not to physiotherapy. A transient pustular rash on the palms had occurred one year previously.

Examination revealed tenderness to pressure in the region of the left trapezius muscle and pain on percussion of the upper dorsal spine. Cervical spondylosis was suspected. Blood tests showed a slight anemia (Hb $10.9 \mathrm{~g} \%$ ), raised ESR (56 and $99 \mathrm{~mm} / \mathrm{h}$ ), and elevated alpha- 2 and beta globulins. Radiographs showed a mixed lytic and sclerotic lesion of the left second rib with irregular periosteal new bone formation. There was a soft tissue mass in the mediastinum and the apex of the left lung (Fig. 13).

CT showed pleural thickening and a soft tissue mass adjacent to the left second rib extending into the posterosuperior mediastinum (Fig. 1C). CT detected additional areas of bony sclerosis in the left first rib, and the first and second thoracic vertebral bodies. The patient was treated conservatively with a nonsteroidal anti-inflammatory (ibuprofen $400 \mathrm{mg}$ ) with good initial results, but relapsed after 3 months with reappearance of the palmar pustules.

\section{Discussion}

Intersternocostoclavicular hyperostosis is a recently recognized disorder characterized by sclerosis and hyperostosis of the sternum, clavicles, and upper ribs together with ossification of the surrounding soft tissues $[9,12]$. Most patients with this disease are in their fifth and sixth decades.

Chronic recurrent multifocal osteomyelitis is a similar disease, characterized by thoracic hyperostosis. This disease affects children and young adults and the sclerotic lesions are often more widespread. Both intersternocostoclavicular hyperostosis and chronic recurrent multifocal osteomyelitis are associated with palmoplantar pustulosis in about $50 \%$ of cases, and spondylitis and/or sacroiliitis in about $40 \%$ of cases $[1,5,11,12]$. In this series, all eight patients had episodic palmoplantar pustulosis and four $(50 \%)$ had spondylitis/ sacroiliitis (Table 1). 

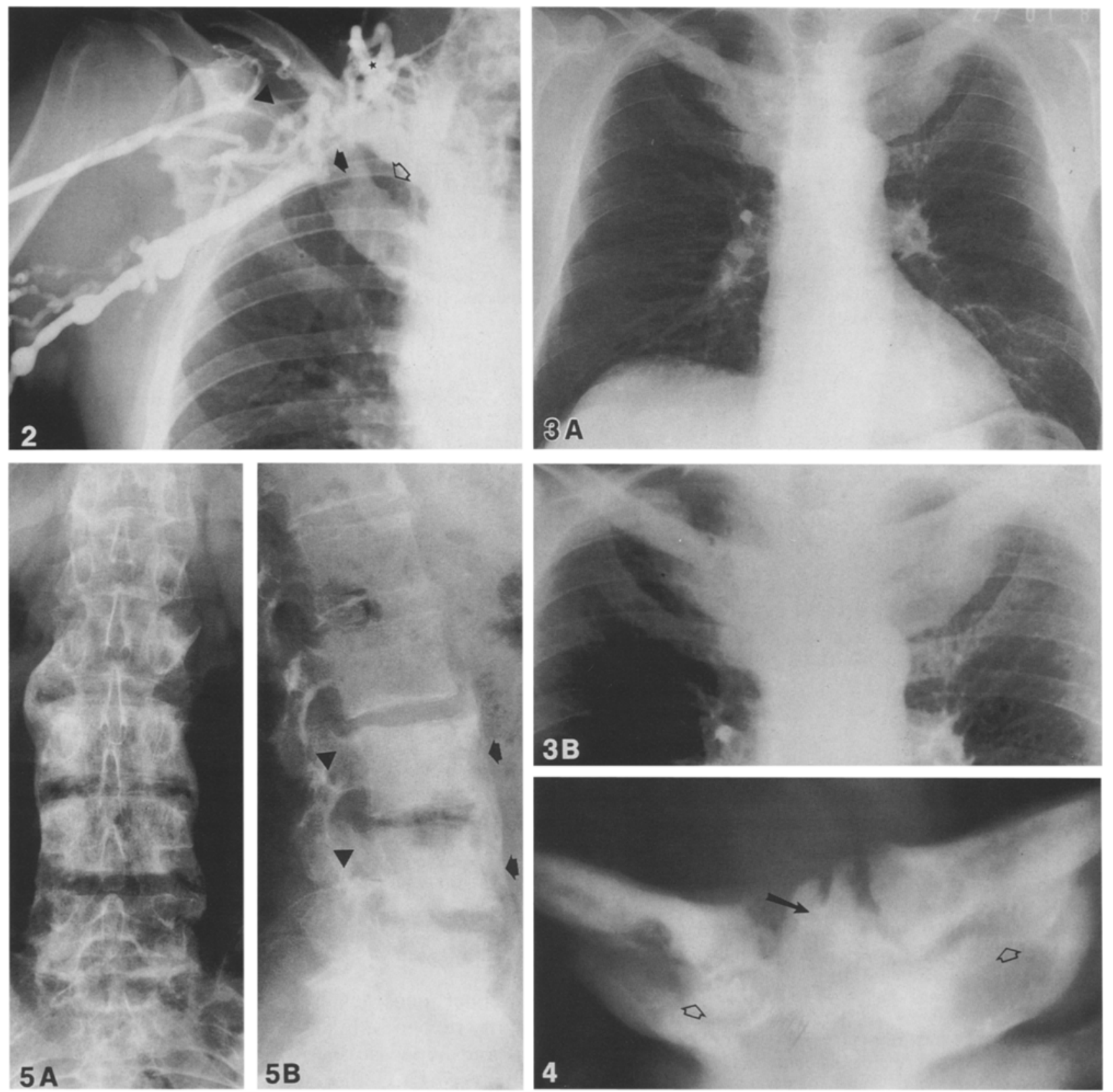

Fig. 2. Case 1. Right upper arm venogram. There is abrupt occlusion of the right subclavian (black arrow) and cephalic veins (black arrowhead), a finding consistent with thrombosis. There are collateral veins in the neck (asterisk). The right 1st and 2nd rib and the medial end of the right clavicle were most affected by sclerosis and hypertrophy. The thoracic inlet is open (open arrow)

Fig. 3. Case 2. Admission chest film of a patient with atypical retrosternal pain shows hyperostosis and sclerosis of both clavicles and the 1 st and 2 nd ribs. There is synostosis between these ribs and the sternum

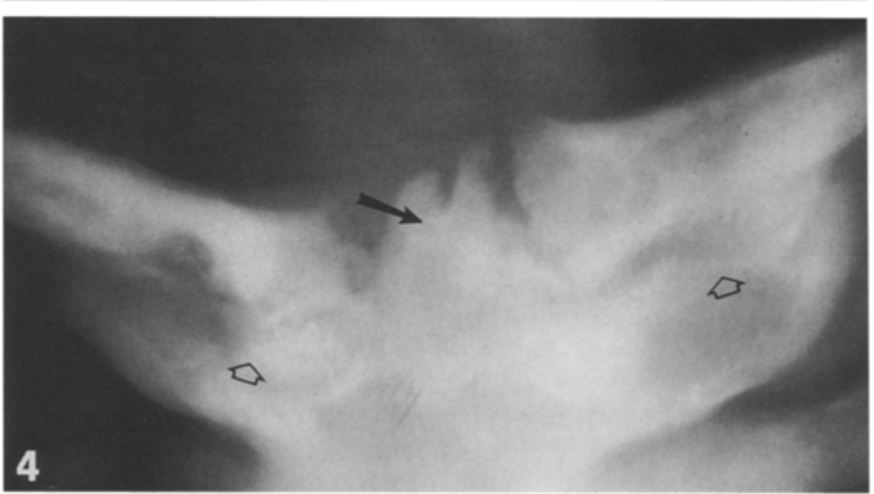

Fig. 4. Case 2. Frontal tomogram of the sternoclavicular junction. There is bony hypertrophy of clavicles, ribs, and sternum and ossification of the sternomastoid tendon insertion (arrow) and costoclavicular ligaments (open arrows)

Fig. 5. Case 3. A AP view of the lumbar spine showing bridging syndesmophytes. The vertebral bodies are enlarged and have a coarse trabecular pattern with areas of sclerosis. B Lateral view of the lumbar spine showing facet joint ankylosis (arrowheads) at multiple levels, hyperostotic and dense lower lumbar vertebrae, and hypertrophic ossification of the anterior longitudinal ligament (arrows). Disc space narrowing with ankylosis occurs at all levels 

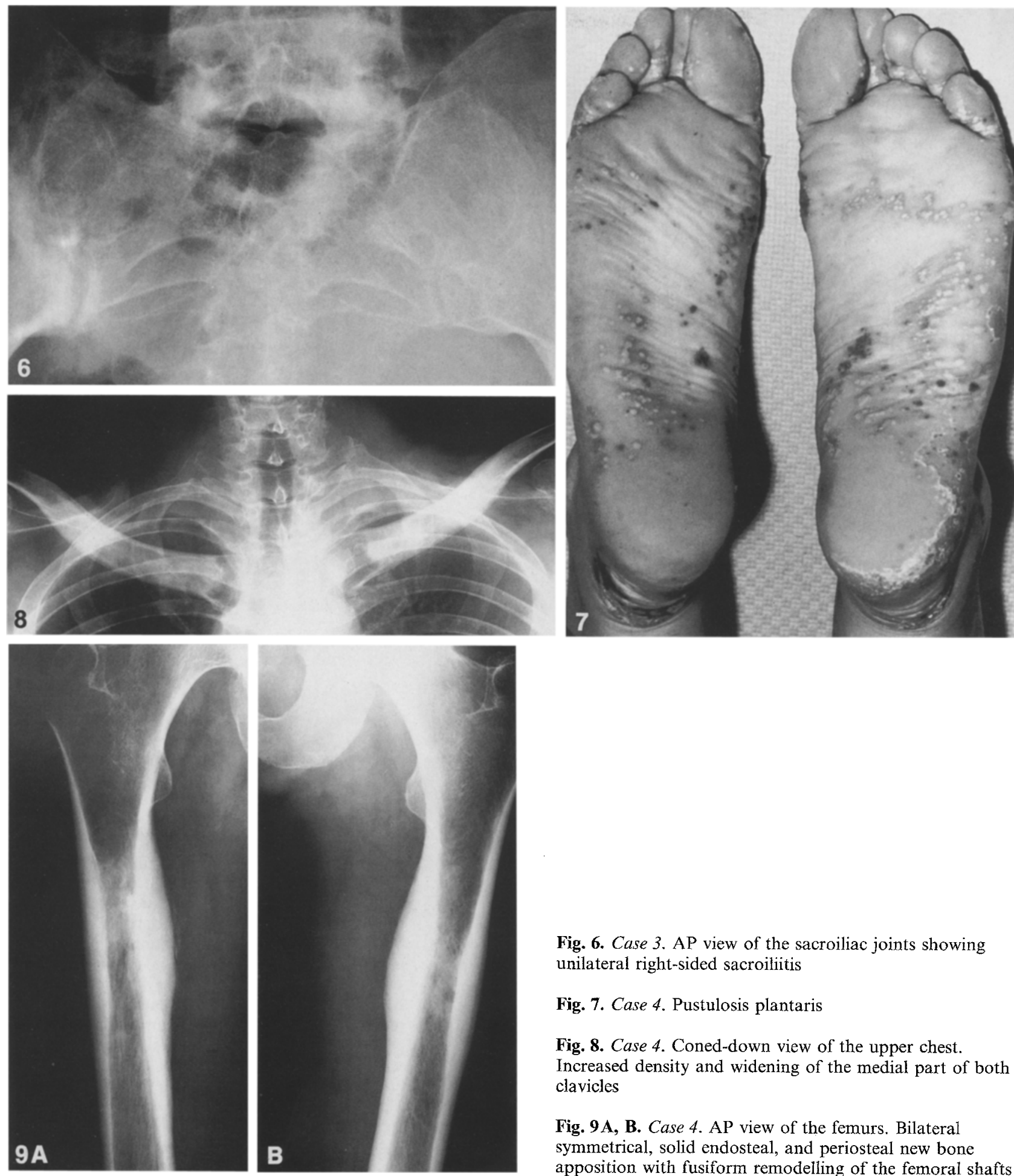

Fig. 6. Case 3. AP view of the sacroiliac joints showing unilateral right-sided sacroiliitis

Fig. 7. Case 4. Pustulosis plantaris

Fig. 8. Case 4. Coned-down view of the upper chest. Increased density and widening of the medial part of both clavicles

Fig. 9A, B. Case 4. AP view of the femurs. Bilateral symmetrical, solid endosteal, and periosteal new bone apposition with fusiform remodelling of the femoral shafts

The sacroiliitis in both syndromes is typically unilateral [1] (Fig. 6), and arthritis is present in $10 \%$ of cases [1]. Periostitis of long bones has also been described $[1,2,5]$. One of our patients had arthritis of the wrists and periostitis of the femora and the bones of the forearms (Fig. 9). Although the bone manifestations of chronic recurrent multifocal osteomyelitis and intersternocostoclavicular hyperostosis are well described $[2,7,9-12]$, it is not well known that many cases have inflammato- 

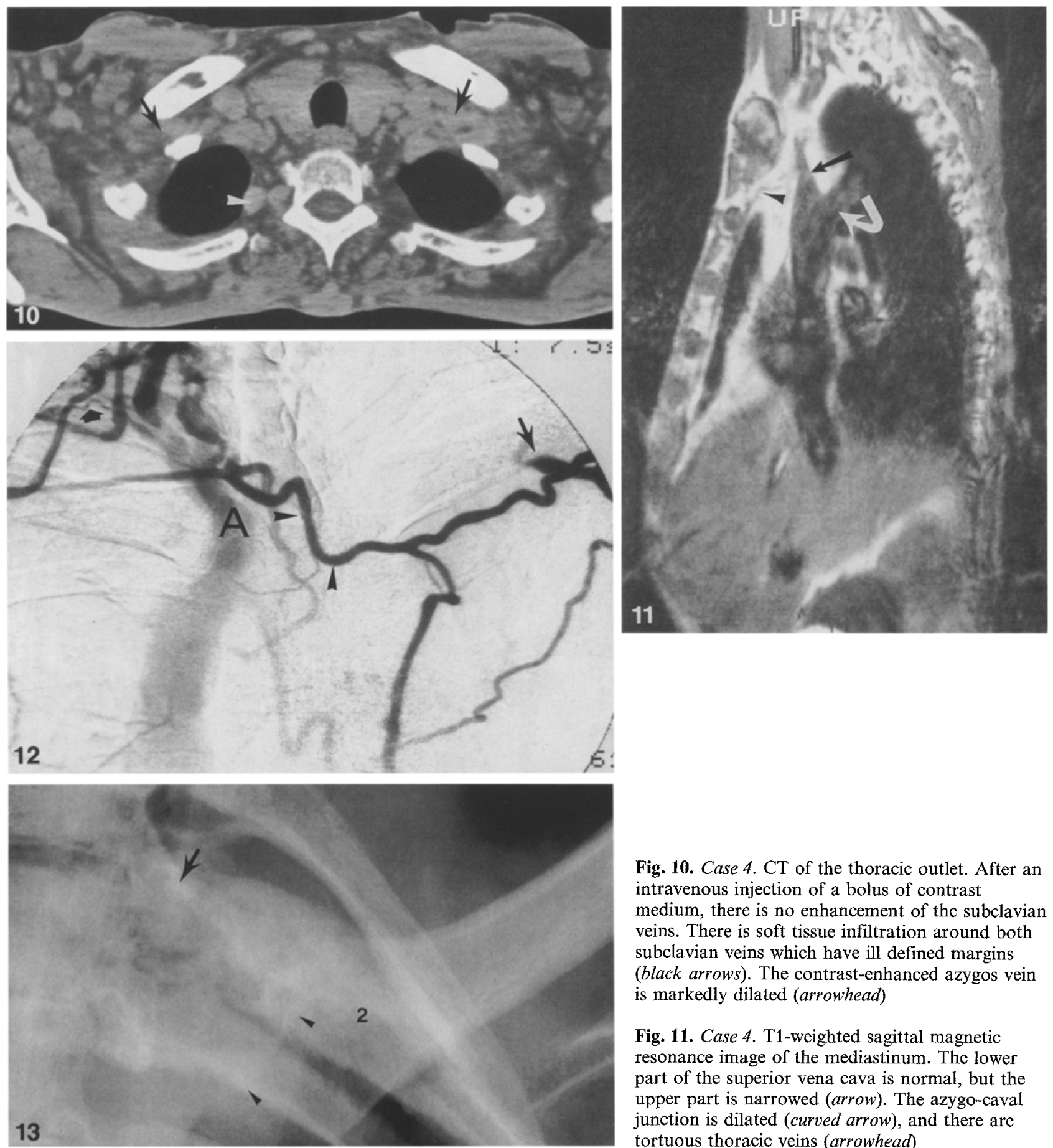

Fig. 10. Case 4. CT of the thoracic outlet. After an intravenous injection of a bolus of contrast medium, there is no enhancement of the subclavian veins. There is soft tissue infiltration around both subclavian veins which have ill defined margins (black arrows). The contrast-enhanced azygos vein is markedly dilated (arrowhead)

Fig. 11. Case 4. T1-weighted sagittal magnetic resonance image of the mediastinum. The lower part of the superior vena cava is normal, but the upper part is narrowed (arrow). The azygo-caval junction is dilated (curved arrow), and there are tortuous thoracic veins (arrowhead)

Fig. 12. Case 4. Oblique view with digital subtraction of the upper chest after simultaneous injection of both antecubital veins. No contrast medium is seen in the subclavian veins, and there is abrupt termination of the left axillary vein (black arrow). Contrast injected in the right arm drained via collateral veins in the right neck into hypertrophied intercostal veins (small black arrow) and dilated azygos system $(A)$. Contrast injected into the left arm passed sequentially into large, tortuous intercostal veins, the accessory hemiazygos vein (arrowheads), and the azygos vein $(A)$

Fig. 13. Case 5. Detailed view of the ribs in the left upper chest wall. There is hypertrophy of the 2nd rib (2) and associated pleural thickening and mediastinal widening (arrowheads). There are coarse trabeculae in the vertebral bodies at T1 and T2, and the left transverse process (arrow) of $\mathrm{T} 2$ is enlarged (arrow) 
Table 1. Some characteristics of the patients: Case, number and sex

\begin{tabular}{|c|c|c|c|c|c|c|c|c|}
\hline & 1 & 2 & 3 & 4 & 5 & 6 & 7 & 8 \\
\hline & M & $\mathrm{M}$ & $\mathrm{F}$ & M & $\mathrm{F}$ & $\mathrm{F}$ & $\mathrm{M}$ & $\mathrm{M}$ \\
\hline Age at onset disease (years) & 46 & 53 & 39 & 43 & 21 & 41 & 49 & 49 \\
\hline Pustulosis palmoplantaris & once & once & once & recurrently & recurrently & once & recurrently & recurrently \\
\hline Number of open biopsies & 2 & 1 & - & - & - & 2 & 3 & - \\
\hline $\begin{array}{l}\text { Hyperostotic lesions: } \\
\text { sternum } \\
\text { clavicles } \\
\text { ribs }\end{array}$ & $\begin{array}{l}- \\
\text { asymmetric } \\
\text { right } \\
1 \text { st, } \\
2 \text { nd ribs }\end{array}$ & $\begin{array}{l}+ \\
\text { symmetric } \\
\text { symmetric } \\
1 \text { st, } \\
\text { 2nd ribs }\end{array}$ & $\begin{array}{l}+ \\
\text { asymmetric } \\
\text { symmetric } \\
\text { 1st rib }\end{array}$ & $\begin{array}{l}- \\
\text { symmetric } \\
\text { symmetric } \\
\text { 1st rib }\end{array}$ & $\begin{array}{l}- \\
- \\
\text { left } \\
1 \text { st, } \\
\text { 2nd ribs }\end{array}$ & $\begin{array}{l}+ \\
\text { asymmetric } \\
\text { symmetric } \\
1 \text { st rib }\end{array}$ & $\begin{array}{l}+t \\
\text { asymmetric } \\
\text { symmetric } \\
\text { 1st rib }\end{array}$ & $\begin{array}{l}+ \\
\text { symmetric } \\
\text { symmetric } \\
\text { 1st rib }\end{array}$ \\
\hline Manubrio-sternal arthritis & - & + & + & - & - & + & + & + \\
\hline $\begin{array}{l}\text { Ossification costo- } \\
\text { clavicular ligaments }\end{array}$ & right & bilateral & left & bilateral & - & bilateral & left & bilateral \\
\hline $\begin{array}{l}\text { Spondylitis, sclerosis } \\
\text { of vertebrae and vertebral } \\
\text { ligaments }\end{array}$ & - & - & + & ++ & + & - & $\ldots$ & ++ \\
\hline Hyperostosis long bones & - & - & - & ++ & - & - & - & - \\
\hline Sacro-iliitis & - & - & unilateral & unilateral & - & - & - & unilateral \\
\hline Venous thrombosis & right & - & - & bilateral & - & - & - & - \\
\hline $\begin{array}{l}\text { Mediastinal or pleural } \\
\text { involvement with } \\
\text { extraosseous soft tissue } \\
\text { infiltration }\end{array}$ & ++ & ++ & $\mathrm{N}$ & + & ++ & + & + & + \\
\hline $\begin{array}{l}\text { B27 } \\
\text { RA factor and Waaler Rose }\end{array}$ & & & - & \multicolumn{5}{|c|}{ - Negative for all patients } \\
\hline
\end{tabular}

$\mathrm{N}=$ No information, no $\mathrm{CT}$ scan performed; $++:$ Extensive $;:$ Minor

ry soft tissue involvement, sometimes with venous obstruction $[4,6,8]$.

Seven of the eight patients in this series had CT examinations of thoracic lesions which showed soft tissue involvement of the mediastinum and thoracic wall. In three patients this involvement was severe (Fig. $1 \mathrm{~A}-\mathrm{C}$ ), simulating a tumor in one (Fig. 1A). The other patients had only minimal periosseous soft tissue infiltration (Fig. 1D). Furthermore, there were hyperostotic lesions of entheses in seven of eight patients (Fig. 4).

These cases show that the soft tissue lesions in pustulotic artho-osteitis may be conspicuous and may be misdiagnosed as neoplasms. This misdiagnosis occurred in three cases. One patient (case 7) had three open bone biopsies because of suspected sarcoma complicating Paget disease. In another patient (case 1), a parasternotomy and several biopsies were performed for a restrosternal pseudotumor which had caused unilateral thoracic edema. Patient 5 presented with soft tissue swelling over the dorsal aspect of the thorax for which the surgeon considered a rib resection. The plain films and CT showed hyperostosis involving three verte- brae and two adjacent ribs and extensive periosseous soft tissue swelling. This extensive involvement and the radiological diagnosis of chronic recurrent multifocal osteomyelitis restrained the surgeon from performing a mutilating operation (Fig. 1C).

Varied symptoms and nonspecific laboratory findings $[3,11]$ are additional reasons for misdiagnosis. Retrosternal pain in one patient (case 2), initially misinterpreted as angina pectoris, led to coronary arteriography. The atypical pain resolved after administration of an anti-inflammatory agent. Two of our patients (cases 6 and 8) had been treated for nonspecific muscular pain in the shoulder and neck.

Venous thrombosis in patients with thoracic hyperostosis and palmoplantar pustulosis was first noted by Kohler et al. [8] and was subsequently mentioned by others $[4,6]$. Most affected patients are white. The thrombosis has been attributed to narrowing of the thoracic outlet by hyperostotic bone. However, the two patients with mediastinal vein thrombosis in this series (cases 1 and 4) had a widely patent thoracic outlet on CT. One of these 
patients had a high level of lupus anticoagulant factor (case 4), and the other had marked perivenous inflammation. The significance of the lupus anticoagulant factor is not known. It is found in several autoimmune diseases and in association with widespread veno-occlusive disease and retroperitoneal fibrosis (as in case 4). Gerster et al. proposed an autoimmune pathogenesis of pustulotic arthro-osteitis in a case report of a patient with concurrent vasculitis.

In our two cases of thrombosis of the innominate veins, a subcutaneous venous collateral network was seen, and there was marked edema of the upper thorax. The tentative clinical diagnosis in these cases was superior vena cava obstruction by mediastinal tumor mass. Exploratory surgery was performed in one case. It is not clear why this disease should preferentially affect large mediastinal veins and the veins of the thoracic outlet in the absence of thoracic outlet obstruction, nor is it clear whether a disturbance of coagulation is an etiologic factor.

There are several causes of cortical hyperostosis including trauma, vitamin A poisoning, and pulmonary osteoarthropathy, but none of these is responsible for the chest hyperostosis seen in chronic recurrent multifocal osteomyelitis and intersternocostoclavicular hyperostosis $[7,12]$. The association of both diseases with recurrent respiratory infections is unexplained [7, 12]. An immune deficiency and an autoimmune reaction to bone have both been proposed as causes, but no definite evidence for either has been provided [7].

An extensive search for bacteria which included aerobic and anaerobic bacterial cultures from blood and bone biopsy material proved negative in our cases and in those reported in the literature. Several authors report negative cultures for $\mathrm{Myco}$ bacterium tuberculosis and fungi $[2,5,8]$. Also, serological tests for bacteria and viruses are normal. These findings indicate a noninfectious cause even though the white cell count is often raised in these patients.

Infiltration by plasmocytes has recently been suggested to explain new bone production in a variety of conditions including chronic recurrent multifocal osteomyelitis and intersternocostoclavicular hyperostosis [11]. Only a few authors, how- ever, mention the presence of plasmocytes in their biopsy material, and the biopsies in four of our patients showed chronic inflammation without plasmocytes as a feature. Several authors classify pustulotic artho-osteitis as a seronegative spondyloarthropathy claiming that it has much in common with this group of diseases, particularly absent rheumatoid factor, sacroiliitis, peripheral inflammatory arthritis, thrombophlebitis, and skin lesions $[1,6,11,12]$.

The etiology and pathogenesis of pustulotic arthro-osteitis are still obscure [11]. Nonetheless, clinicians and radiologists should be aware of this inflammatory disease because it can cause a tumorlike mediastinitis with thrombosis of mediastinal and subclavian veins.

\section{References}

1. Beraneck L, Kaplan G, Benoist M, Bauchon JP, Prost A, Vassal JP, Kahn MF (1984) Hyperostose multiple avec sacro-iliite unilaterale. Une nouvelle spondylarthropathie. Presse Med 13:2001

2. Bjorksten B, Gustavson K, Eriksson B, Lindholm A, Nordstrom $S$ (1978) Chronic recurrent multifocal osteomyelitis and pustulosis palmoplantaris. J Pediatr 93:227

3. Gerster JC, Lagier R, Nicod L (1985) Case report 311. Skeletal Radiol 14:53

4. Hoenel LC, Bradway WR, Constantini PJ (1980) Thrombophlebitis complicating sternocostoclavicular hyperostosis. Postgrad Med 68:113

5. Huaux JP, Maldague B, Malghem J, Meunier H, Noel H, Nagant-de-deux Chaisnes C (1986) Pustulotic arthroosteitis and related disorders in children and adults. (A report of seven cases.) J Belge Radiol 69:345

6. Jirik FR, Stein HB, Chalmers A (1982) Clavicular hyperostosis with enthesopathy, hypergammaglobulinemia, and thoracic outlet syndrome. Ann Intern Med 97:48

7. Keipert JA, Campbell PE (1970) Recurrent hyperostosis of the clavicles: an undiagnosed syndrome. Aust Paediatr J 6:97

8. Kohler H, Uehlinger E, Kutzner J, West TB (1977) Sternocostoclavicular hyperostosis: painful swelling of the sternum, clavicle, and upper ribs. Ann Intern Med 87:192

9. Resnick D (1980) Sternocostoclavicular hyperostosis. AJR 135: 1278

10. Resnick D, Vint V, Poteshman NL (1981) Sternocostoclavicular hyperostosis. J Bone Joint Surg [Am] 63:1329

11. Sartoris JD, Schreiman JS, Kerr R, Resnik CS (1986) Sternocostoclavicular hyperostosis: a review and report of 11 cases. Radiology 158:125

12. Sonozaki $H$, Mitsui $H$, Miyanaga $Y$, Okitsu $\mathrm{K}$, Igaraski M, Hayashi Y, Matsuura M, Azuma A, Okai K, Kawashima M (1981) Clinical features of 53 cases with pustulotic arthro-osteitis. Ann Rheum Dis 40:547 